\title{
Holistic Care: An Urgent Need for Cervical Cancer Patients in Kenya
}

\author{
Jane Adhiambo Owenga \\ Department of Public Health and Community Health and Development, School of Health Sciences, Jaramogi Oginga Odinga University of \\ Science and Technology, Bondo, Kenya
}

Email address:

adhiambojane33@yahoo.com

\section{To cite this article:}

Jane Adhiambo Owenga. Holistic Care: An Urgent Need for Cervical Cancer Patients in Kenya. International Journal of European Studies. Vol. 3, No. 1, 2019, pp. 15-22. doi: 10.11648/j.ijes.20190301.13

Received: February 28, 2019; Accepted: April 3, 2019; Published: May 6, 2019

\begin{abstract}
Holistic health care is an integrated approach to healthcare that treats the whole person, not simply symptoms and disease. Mind and body are integrated and inseparable. In cancer care, this approach has been actualized through the implementation of palliative care. The study aimed to determine holistic care issues faced by cervical cancer patients in Kenya. A cross-sectional study involving 334 cervical cancer patients was conducted in Jaramogi Oginga Odinga Teaching and Referral Hospital (JOOTRH) in Kisumu from September 2014 to February 2015. Structured questionnaire, in-depth interview guide and key informant interview guide were used to collect data. Quantitative data was analyzed using Statistical Package for Social Scientists (SPSS) Version 20 at a statistical significance of $\mathrm{P} \leq 0.05$, descriptive statistics were performed. For qualitative data, the responses were transcribed verbatim and the content was then analyzed by searching for emerging themes on holistic care issues faced by cervical cancer patients. Qualitative data was presented in textual form with verbatim reports for illustrations. The key holistic care issues from the study were being bothered by discharge, bleeding and odor from vagina $260(78 \%)$, being afraid to have sex $283(85 \%)$, lack of access to counseling $314(87 \%)$, minimal acceptance of their disease by family members 263 (79\%), need for prayers from spiritual members $313(94 \%)$ and having pain 324 (97\%). The study concluded that cervical cancer patients are a facing a myriad of holistic care issues. Therefore there is a need for the ministry of health to develop interventions that can cushion them from such issues.
\end{abstract}

Keywords: Holistic Care, Cervical Cancer, Kenya

\section{Introduction}

Holistic health care is an integrated approach to healthcare that treats the whole person, not simply symptoms and disease. Mind and body are integrated and inseparable. In cancer care, this approach has been actualized through the implementation of palliative care. Palliative care is an approach that improves the quality of life of patients and their families facing the problems associated with lifethreatening illness, through the prevention and relief of suffering by means of early identification and impeccable assessment and treatment of pain and other problems such as physical, psychosocial and spiritual concerns [1]. In the developed world palliative care has been intergrated in the healthcare system and has well established policies that guide its practice and resource allocation for its implementation [2].

Palliative care in Africa is still at its initial stages with onlytwo out of 53 countries (Rwanda, Swaziland) having a stand-alone national policy, strategic and implementation plan. While only four countries (Uganda, Kenya, Tanzania, South-Africa) have integrated palliative care into their public health services [3,4]. The little focus on palliative care witnessed in Africa may be due to the current double burden of disease where the healthcare system is grappling with communicable diseases and rising cases of noncommunicable diseases with little resource base. In situations where palliative care is practiced in Africa, focus has been more on HIV and AIDs patients with little on cancer patients in general without special emphasis on individual site specific cancers. This missing link can be filled as different types of cancers are faced with unique problems that require specialized attention.

Palliative care in Kenya was officially launched in the year 2012-Cancer Prevention and Control Act 2012 [5]. However, 
it is still facing myriad of challenges including inadequate experts and limited resources. Therefore, its implementation is not well inscribed in the Kenyan health provision system [6]. According to the Kenya human rights charter, patients have a right to participate in decision making and designing healthcare services appropriate for them. They also have a right to complain about healthcare services that they consume. This requirement so far is not provided for in Kenyan palliative care, there is no evidence that cervical cancer patients have been given an opportunity to participate in articulating their palliative care needs.

There is evidence of unmet psychosocial, physical, financial and spiritual needs among cancer patients in Uganda, South Africa, Tanzania and Ethiopia mainly due to inadequate experts and limited resources $[3,4,7,8]$. No such study has specifically focused on cervical cancer patients in western Kenya.

This study was conducted in western Kenya, Jaramogi Oginga Odinga Teaching and Referral hospital. This hospital is the referral facility for all cancer patients from peripheral facilities in western Kenya. Much as cervical cancer prevalence in western Kenya is not exactly known due to lack of functional cancer registry in this region, it is suspected to be among the highest in Kenya based on the fact that, Western Kenya has some of the highest HIV and AIDS prevalence at 15.1 percent which is double the national HIV prevalence of 5.6 percentUNAIDS [9] and KAIS [10], and cervical cancer is highly associated with HIV/AIDS [11].

\section{Methods}

\subsection{Study Setting}

The study was conducted at JOOTRH in Kisumu from September 2014 to February 2015. JOOTRH is a referral hospital serving a catchment area with a population of more than 5 million people in more than 10 counties in the western region of Kenya. The hospital serves an area with some of the worst health indicators in the country including high prevalence of HIV infection (15.4\%) which is greater than twice that of the national (7.1\%) prevalence [12]. JOOTRH is the referral hospital for cancer patients in western part of Kenya. At the time of this study, total in-patient cervical cancer admissions were 681 and 735 in 2014 and 2015 respectively.

\subsection{Study Design}

This was a hospital based cross-sectional study. It involved collection of both quantitative and qualitative data from cervical cancer patients seeking care at JOOTRH.

\subsection{Study Participants}

The study participants consisted of cervical cancer patients who were over 18 years visiting JOOTRH or referred to JOOTRH for further treatment or palliative care services. The eligible respondents were sourced from palliative care clinics, oncology unit, and obstetric and gynecological unit within JOOTRH.

\subsection{Sampling Design}

The sampling strategy involved purposive sequential enrolment of patients with histologically proven cervical cancer as they became available at the facility till the required sample size was reached. Healthcare workers were also purposively sampled for key informant interview. Only those who consented were sampled. The healthcare providers in charge of the patients and palliative care specialist helped identify patients based on information in the patient files then referred them to the researcher who confirmed their eligibility and proceeded to seek consent from each of them. This exercise was done every day in the gynecological ward (ward 4) and in room 19/16 where cervical cancer screening, cryotherapy and LEEP was performed. Patients were also selected on Tuesdays in the gynecological outpatient clinics (GOPC) where patients who were diagnosed at early stage were done for surgery. This was done until the desired sample size (334) was achieved. Participants for in-depth interviews were selected by the care providers based on how long they had been symptomatically sick (at least more than one year) and consented to the study. Pilot survey was conducted at the neighboring Kisumu East sub-county hospital to test the study tools and improve their quality and efficiency.

\subsection{Research Procedure}

A structured questionnaire was administered to the recruited patients by the researcher and research assistants. The researcher liaised with the relevant health care providers at the palliative care unit, obstetric/gynecological wards and on GOPC days which were appropriate for identification of the eligible clients. The researcher and the health care team worked out a programme on how the researcher could access the respondents without putting any strain on the respondents such as by keeping them longer in the facility. The researcher also took contacts of clients who consented to the study but were not able to respond to the questionnaire at that time and made private arrangement with them on when and where it was convenient to administer the questionnaire to them. The participants answered the questionnaires by themselves except for some $21(6.3 \%)$ who needed help and were assisted by the researcher.

Four healthcare workers were interviewed by the researcher face to face and their responses were audio recorded and transcribed verbatim. In-depth interview guide was used to collect qualitative data from 12 eligible patients by the researcher. The patients were guided to narrate their story concerning their wellbeing challenges during the disease trajectory. Both sessions took about 40 minutes each. They were audio recorded and later transcribed verbatim and content analysis done and themes generated to enrich quantitative data. Finally, the transcripts were returned to the healthcare workers and 7 patients ( 5 of them had died) who were in agreement that the information reflected their 
expressions.

\subsection{Data Management and Analysis}

Quantitative data was coded, edited and cleaned to check for any errors and entered in Statistical Package for Social Scientists (SPSS) version 20 and presented in tables.

For qualitative data, the responses were transcribed verbatim; the content was then analyzed by searching for emerging themes on the wellbeing challenges faced by cervical cancer patients. Qualitative data was presented in textual form with verbatim reports alongside quantitative data for illustrations.

\subsection{Ethical Consideration}

Ethical clearance was obtained from JOOTRH Ethical Review Board, reference number- (ERC.1B/VOL.1/135). The nature and purpose of the study was explained to the potential participants verbally and in writing (if the patient could read) to enable them make informed consent as a basis for enrollment. Participation was voluntary, confidentiality anonymity was guaranteed. Individual written consent was obtained from each participant before being enrolled into the study. Patients who were unable to write were asked to sign through thumb stamping.

\subsection{Quality Assurance}

Pilot survey was conducted at the neighboring Kisumu East sub-county hospital. Content validity was assessed and approved by palliative care team at Kisumu hospice who are competent and experienced in quality of life issues.

\section{Results}

\subsection{Socio-Demographic Characteristics of Respondents}

A total of 334 cervical cancer patients participated in the study. A larger proportion 114 (34\%) were aged between 3646 years. More than half (53\%) were widowed, and only 30 (9\%) were formally employed.

Table 1. Socio-Demographic Characteristics of Respondents.

\begin{tabular}{llc}
\hline Characteristics & Categories & n (\%) \\
\hline Age group & & \\
& $18-35$ & $93(28)$ \\
& $36-46$ & $114(34)$ \\
& $47-57$ & $52(16)$ \\
& 58 and above & $75(22)$ \\
Marital status & Married & $104(31)$ \\
& Divorced/separated & $31(10)$ \\
& Widowed & $178(53)$ \\
& Never married/ single & $21(6)$ \\
Level of education attained & None & $43(13)$ \\
& Primary & $179(54)$ \\
& Secondary & $62(18)$ \\
& Tertiary & $50(15)$ \\
Religion & Christians & $324(97)$ \\
\hline
\end{tabular}

\begin{tabular}{lll}
\hline Characteristics & Categories & n (\%) \\
\hline \multirow{2}{*}{ Family's main source income } & Muslims & $10(3)$ \\
& Small scale farming & $159(48)$ \\
& Small scale business & $145(43)$ \\
& Formal employment & $30(9)$ \\
Total & & $334(100)$ \\
\hline
\end{tabular}

Key:

None- respondent did not undergo formal education at all.

Primary-respondent attained primary education of any level.

Secondary- respondent attained secondary education of any level.

Tertiary - respondent attained any training post-secondary education.

\subsection{Clinical Characteristics}

One seventy-nine $(54 \%)$ of the respondents were at stage IV, and $63(19 \%)$ at stage III.

Most of the respondents (63\%) were (HIV) Positive while only $73(22 \%)$ were negative.

One hundred and five $(31 \%)$ of the patients were treated by blood transfusion and use of pain killers while only (9\%) of the patients were treated by surgery and $42(13 \%)$ by LEEP.

Table 2. Clinical Characteristics of Respondents.

\begin{tabular}{lll}
\hline Characteristics & Categories & n (\%) \\
\hline Cervical cancer stage & Stage I & $52(15)$ \\
& Stage II & $40(12)$ \\
& Stage III & $63(19)$ \\
& Stage IV & $179(54)$ \\
Cervical cancer screening & Yes & $112(33)$ \\
& No & $222(67)$ \\
HIV status & & \\
& HIV Positive & $209(63)$ \\
& HIV Negative & $73(22)$ \\
Period of diagnosis & Don't know & $52(15)$ \\
& & \\
& Less than a year ago & $217(65)$ \\
& More than a year ago & $31(9)$ \\
Treatment received & Don't know & $86(26)$ \\
& & \\
& Chemotherapy & $32(10)$ \\
& Radiotherapy and chemotherapy & $11(3)$ \\
& Total hysterectomy & $30(9)$ \\
& Blood transfusion and pain killing & $105(31)$ \\
& Pain killing only & $74(22)$ \\
& Haematemics & $40(12)$ \\
& Conization (LEEP) & $42(13)$ \\
& & $334(100)$ \\
\hline
\end{tabular}

\subsection{Psychological Issues of Cervical Cancer Patients}

Most of the respondents were very much bothered by bleeding, discharge and or odor from vagina $260(78 \%)$ with only $74(22 \%)$ a little bit bothered. More than half of the respondents $220(66 \%)$ were a little bit worried about death with $61(18 \%)$ quite a bit worried and $53(16 \%)$ very much worried about death.

A large proportion of respondents $283(85 \%)$ were very much afraid to have sex with other $41(12 \%)$ quite a bit afraid and only $10(3 \%)$ a little bit afraid to have sex. Only $20(6 \%)$ 
of the patients stated that they were seriously loosing hope in the fight against their illness with most patients $284(85 \%)$

only slightly loosing hope. Table 3 summarizes the results.

Table 3. Psychological issuesof Cervical Cancer Patients.

\begin{tabular}{llll}
\hline Psychological issues & A Little bit n (\%) & Quite a bit n (\%) & Very much n (\%) \\
\hline Bothered by discharge/ bleeding/ odor from my vagina & $74(22 \%)$ & $0(\%)$ & $260(78 \%)$ \\
Worry about dying & $220(66 \%)$ & $61(18 \%)$ & $53(16 \%)$ \\
Afraid to have sex & $10(3 \%)$ & $41(12 \%)$ & $283(85 \%)$ \\
I am losing hope in the fight against my illness & $284(85 \%)$ & $30(9 \%)$ & $20(6 \%)$ \\
\hline
\end{tabular}

In order to further explore the psychological concerns of cervical cancer patients, in- interviews were held with some patients who had been ill for more than one year.

From the in-depth interviews, a theme of low self-esteem emerged. This meant that cervical cancer patients experienced lowered self-worth as a result of disrupted body image brought about by smelling discharge from the patient's vagina. A patient stated that:

I bleed a lot and when bleeding starts it goes even for six hours nonstop and when it stops I get so tired that I even faint, this has made me develop severe anaemia. This is usually followed by dirty creamish, discharge with a bad odour. This makes me feel unclean and lowers my self-esteem so much so that I don't feel like interacting with people. When I travel in public transport coming to the hospital those who sit next to me even hold their noses. I feel like the ground should swallow me so that I don't inconvenience people this much. I am facing a great challenge of keeping clean and hygienic. (37 year old cervical cancer patient).
In an in-depth interview a patient stated that:

I feel a lot of pain during sexual intercourse, and bleed a lot after sex. This has made me develop intense fear for sex until I no longer feel like sex. I have them freed my Husband to marry or seek Sexual satisfaction elsewhere to preserve my health (37 year old cervical cancer patient).

\subsection{Supportive Concerns of Cervical Cancer Patients}

Most patients did not have access to professional counseling at all $314(87 \%)$, while $10(3 \%)$ received very much professional counseling. Majority 273 (81\%) of the respondents reported not counseled on sexual matters at all, while only $10(3.0 \%)$ reported receiving very much sexual counseling. Only 112 (34\%) of the patients reported being sufficiently treated with dignity while 138 (41\%) were not treated with dignity at all. As regards support groups, 314 (94\%) did not have access to support groups at all with 20 (6\%) having access to support groups. Table 4 illustrates the results.

Table 4. Supportive concerns of Cervical Cancer Patients.

\begin{tabular}{|c|c|c|c|}
\hline Supportive concerns & None at all n (\%) & Quite a bit n (\%) & Very much n (\%) \\
\hline Access to professional counseling & $314(87 \%)$ & $10(3 \%)$ & $10(3 \%)$ \\
\hline Counseled on sexual matters & $273(81 \%)$ & $11(3 \%)$ & $20(6 \%)$ \\
\hline Treated with dignity & $138(41 \%)$ & $84(25 \%)$ & $112(34 \%)$ \\
\hline Access to support groups & $314(94 \%)$ & $0 \%$ & $20(6 \%)$ \\
\hline
\end{tabular}

It was in the interest of the study to further explore supportive needs of cervical cancer patients from HCWs. From the interviews with healthcare workers a theme of lack of funding emerged. This meant that there was no funding to support the activities of the support groups; a palliative care nurse stated that:

We do try to form support groups for the patients but it has not worked due to lack of support with logistics such as T.V, snacks, meeting space and fare reimbursement. We do not have any funding and that most patients are too sick to attend the meetings and worst of all the patients die quite fast after diagnosis since most of them cannot afford treatments of late stage (HCW6).

Another theme that emerged from interviews with care providers at the palliative care unit was lack of professional counselors. This meant that the facility lacked qualified palliative care counselors. A healthcare provider thus, explained:

We don't have professional counselors in the unit, nowadays VCT counseling have neutralized professional counseling, most providers only attend in- service VCT counseling and are considered qualified......so we need professional counselors for our patients (HCW1).

The study also revealed the theme on lack of sexual counseling. This implies information given to the cervical cancer patients and their spouses by the care providers, with the aim of enabling the patients and their spouses to understand and appreciate the effect that the disease and its treatment have on their sexuality. This is geared towards helping them manage and develop coping strategies that can enable them improve their wellbeing as concerns sexual matters. A patient stated that:

Our culture restricts sexual communication and holds that sexual matters must be handled with utmost secrecy. Worse still, women are only supposed to submit to sexual advances and not talk about sex. More often we are viewed as suffering from Sexually Transmitted Infections' and therefore not clean and are abandoned by our husbands or "inheritors", we are then forced to kill our sex life for lack of alternative (36 year old cancer patient).

\subsection{Social Concerns of Cervical Cancer Patients}

Most of the respondents $221(66 \%)$ reported receiving a little support from their friends with 42 (13\%) receiving quite 
a bit of support from friends while only 60 (18\%) received very much support. More than half 169 (51\%) of respondents felt a little bit close to their friends with $53(16 \%)$ quite abit close to their friends and $112(34 \%)$ very much close to their friends. Almost half 156 (46.7\%) of the respondents got quite a bit of emotional support from their families with 125 (37\%) getting very much emotional support while $53(16 \%)$ getting alittle bit support. Only 20 (6.0\%) were very much satisfied with their family communication about their illness. Most of the respondents reported that their families have a little bit accepted their illness 157 (47\%) with 106 (32\%) reporting that their families have quite abit accepted their illness. On the other hand, $71(21 \%)$ reported that their families accepted their illness very much. Table 5 illustrates this.

Table 5. Social Concerns of Cervical Cancer Patients.

\begin{tabular}{|c|c|c|c|}
\hline Social Concerns & A Little bit n (\%) & quite a bit n (\%) & Very much n (\%) \\
\hline Support from my friends & $221(66 \%)$ & $42(13 \%)$ & $60(18 \%)$ \\
\hline Closeness to friends & $169(51 \%)$ & $53(16 \%)$ & $112(34 \%)$ \\
\hline Family Emotional support & $53(16 \%)$ & $156(47 \%)$ & $125(37 \%)$ \\
\hline Acceptance of my illness & $157(47 \%)$ & $106(32 \%)$ & $71(21 \%)$ \\
\hline Satisfaction with family communication about illness & $178(53 \%)$ & $136(41 \%)$ & $20(6 \%)$ \\
\hline
\end{tabular}

The study further explored social concerns of cervical cancer patients by holding in-depth interviews with some patients. The themes that emerged were inadequate social support and unfavorable communication about the respondents' illness. This meant that the patients did not get adequate social support, and that their family member's communication about their illness was mostly unfavorable. From an in-depth interview a patient reported that:

Our friends, church members and family members visit us and talk with us so we are not so lonely. They also assist us with home and child care. Though, they cannot afford much financial support. Moreover, our illness is not really accepted and that communication about our illness is not pleasant. We tend to be secretive about it since it concerns our private parts and in our culture talking about this is difficult (42 year old cervical cancer patient).

\subsection{Spiritual Wellbeing and Needs of Cervical Cancer Patients}

A large proportion of patients reported finding very much strength and comfort in their spirituality 294 (88\%) with only $20(6 \%)$ finding a little bit strength and comfort in their spirituality. Most patients $272(82 \%)$ very much needed to be visited by their church members, and all patients 334 (100\%) needed very much to listen to gospel music. None of the patients did not express spiritual needs. Table 6 summarizes the results.

Table 6. Spiritual wellbeing and needs of cervical cancer patients.

\begin{tabular}{|c|c|c|c|}
\hline Spiritual wellbeing status and needs & A Little bit n (\%) & Quite a bit n (\%) & Very much n (\%) \\
\hline Finding strength and comfort in spirituality & $20(6 \%)$ & $20(6 \%)$ & $294(88 \%)$ \\
\hline Need visit by church members & $62(18 \%)$ & $0(\%)$ & $272(82 \%)$ \\
\hline Need Prayers from spiritual members & $10(3 \%)$ & $11(3 \%)$ & $313(94 \%)$ \\
\hline
\end{tabular}

Further in-depth interviews with patients were held to explore spiritual status and needs of cervical cancer patients and the theme of finding comfort from prayers emerged. A patient stated that:

I constantly pray and talk to God even in my hospital bed, sometimes people think that I am asleep but just communing with God and praising Him in my heart, I find comfort from that (42 year old cervical cancer patient).

Another theme that emerged was Gods protection. This meant that patients who believed in God were cushioned from worry as they trusted their lives in the hands of God. A patient thus explained:

God has been very good to me this far, I should have died long time ago but due to His protection I am still alive and trust that I will be well soon, He never fails his people (46 year old cervical cancer patient).

\subsection{Physical Issues of Cervical Cancer Patients}

A large proportion of patients 324 (97) reported having very much pain with none having little pain. Similarly, most of the respondents were experiencing very much lack of energy $252(76 \%)$ with $41(12 \%)$ experiencing a little bit lack of energy. Another $213(64 \%)$ were very much bothered by constipation and $191(57 \%)$ had nausea.

Table 7. Physical Issues.

\begin{tabular}{llll}
\hline Physical Issues & A Little bit n (\%) & Quite a bit n (\%) & Very much n (\%) \\
\hline Lack of energy & $41(12 \%)$ & $41(12 \%)$ & $252(76 \%)$ \\
Sleeping well & $42(13 \%)$ & $41(12 \%)$ & $251(75 \%)$ \\
Having pain & $0(\%)$ & $10(3 \%)$ & $324(97 \%)$ \\
Forced to spend time in bed & $52(16 \%)$ & $30(9 \%)$ & $252(75 \%)$ \\
Bothered by constipation & $91(27 \%)$ & $30(9 \%)$ & $213(64 \%)$ \\
Have nausea & $123(37 \%)$ & $20(6 \%)$ & $191(57 \%)$ \\
\hline
\end{tabular}


Further in-depth interview with a patient revealed the theme of fatigue due to anaemia. A patient stated that:

I bleed a lot and when bleeding starts it goes even for six hours nonstop and when it stops I get so tired and fatigued that I even faint, this has made me develop severe anaemia. (39 year old cervical cancer patient).

\section{Discussions}

From the study, majority of respondents reported bleeding, discharge and odor from the vagina, this lowered their selfesteem and impacted negatively on their psychological wellbeing. Most patients were very much afraid to have sex due to pain experienced during coitus leading to low sexual desire and thereby disrupting their social and emotional wellbeing. Other studies have revealed psychological issues among women with gynecological cancers $[6,13,14]$. A large proportion of respondents in this study who were very much afraid to have sex is consistent with Muiva [6], whose finding showed that most respondents in her study on women with gynecological cancers in Kenya expressed sexual function impairment. Other studies have also revealed adverse sexual problems in women with gynecological cancers $[15,16]$. This finding reveals that the respondents' psychosocial wellbeing is disrupted and that they need sexual counseling as well as help to deal with vaginal discharge.

According to this study, running support group activities for cervical cancer patients was difficult due to lack of logistics, this was compounded by patients who were too sick to attend such meetings and most of them died soon after diagnosis. This concurs with qualitative finding which showed that majority of patients did not access support groups. The facility also lacked adequate professional counselors, and that those who were there were mostly VCT counselors who could not competently handle cervical cancer patients psychological needs, this could disrupt their psychological wellbeing. This finding is consistent with other studies that have noted inadequate palliative care experts in Africa $[3,4,7,8]$.

The patients also lacked sexual counseling. This implies information given to the cervical cancer patients and their spouses by the care providers, with the aim of enabling the patients and their spouses to understand and appreciate the effect that the disease and its treatment have on their sexuality. This is geared towards helping them manage and develop coping strategies that can enable them improve their wellbeing as concerns sexual matters. They were viewed as suffering from Sexually Transmitted Infections' and therefore not clean and could be abandoned by their spouses, this led some of them to kill their sexual needs thereby impairing their social and emotional wellbeing.

The lack of professional counseling encountered by the majority of respondents in this study was due to inadequate professional counselors in the facility. The few who received professional counseling may have been beneficiaries of PSC (HIV/AIDS) program that was funded by KEMRI within the facility. Similarly, lack of sexual counseling may be due to lack of professional sexual counselors and sexual counseling protocol in the facility. This finding may also be explained by lack of sexual communication occasioned by culture that restricts sexual communication and holds that sexual matters must be handled with utmost secrecy. Communication in sexual matters is still facing a lot of barriers in our African setting. Aniebue [17], in their report on ethical, socioeconomic, and cultural considerations in gynecologic cancer care in developing countries reiterates that cultural and gender norms precludes women from discussing sexuality issues. Moreover, Lamb [15] stated lack of comfort and difficulty among patients and care providers in handling topic of sexuality.

The finding on lack of well trained counselors in this study supportsRawlinson, Gwyther [18], who identified lack of proper palliative care training among health care providers in Africa.

Report on social concerns in the current study revealed that, most respondents received little social support from their friends and families. This differs with Muiva [6], who reported that social concerns were reasonably met in most patients. This finding may have been occasioned by the difference in palliative care services given in the study settings. Most patients in Muiva [6], were from Kenyatta National Hospital (KNH) and Moi Teaching and Referral Hospital (MTRH) which were the only national hospitals in Kenya and major hospices in Kenya like Nairobi hospice and Eldoret hospice. These facilities are staffed with qualified palliative care professionals including qualified counselors and information materials for cancer patients. Psychosocial interventions offered by these professionals may have empowered the patients and their families to appreciate cancer illness and develop positive attitude towards it. The need for social support displayed in this study among cervical cancer patients supports Betty [19], model which posits social wellbeing as a critical component of quality of life among cancer patients.

This study revealed that majority of the patients found strength and comfort in their spirituality. This confirmed how important spirituality is to patients with chronic conditions such as cervical cancer. Spiritual needs of cancer patients have also been reported by Sepulveda et al. (2003) in their study conducted in a couple of African countries (Botswana, Ethiopia, Tanzania, Uganda and Zimbabwe) on quality of care at the end of life among cancer patients. Similarly, Murray, Grant [2], in their study of dying from cancer in developed and developing countries also reiterated that Kenyan cancer patients were comforted and inspired by belief in God. [7] in their study on Needs of Care and Support of Adult Tanzanians with cancer also noted spiritual needs of cancer patients. Spiritual support from medical team and pastoral care visits have been associated with higher QOL scores near death [20]. The need for spirituality displayed in this study among cervical cancer patients supports Erseket al., 1997 and Betty [19], models which 
posits spirituality as a critical component of wellbeing among cancer patients. This finding shows fairly good spiritual wellbeing and need for spiritual nourishment among this group of patients.

This finding shows spiritual strength among some cervical cancer patients which cushioned them from worrying about death thereby improving their psychological wellbeing. In the quantitative finding, approximately half of the respondents did not worry about death while a few were very much worried about death. This may reflect differences in spiritual strength, as those who are very strong spiritually are bound to see death as a transition to eternal life. Other studies have also revealed improved quality of life as a result of spiritual wellbeing [4, 7]. This finding however contradicts Muiva [6], who reported a large proportion of her respondents losing hope in the fight against their illness and worrying about death. This could be explained by the differences in spiritual strength among the respondents in the studies.

Cervical cancer patients in this study spent a lot of money in maintaining personal hygiene, these involved costs of pads, swabs and medication and other substance that could counter foul smell that emanated from their Vagina. Most patients incurred cost of medication, travel and prescription. In contrast, fewer patients incurred cost of cloths and wigs, and cost of home and child care. This may be explained by strong extended family ties that still exist in this study setting, most patients therefore benefited from free home and child care assistance. While the low number reporting costs incurred in clothes and wigs could be explained by the fact that most patients in this study were not on treatment modalities that interfered with their body, such as hair loss or increase in weight. Such treatments include chemotherapy and radiotherapy, in this study, most patients despite being in stage III\& IV of the disease, were only treated with blood transfusion, haematemics and pain killing. Hence may not have required clothes and wigs. A study conducted in Tanzania on HRQoL and needs of cancer patients also revealed that patients incurred a lot of costs such as medication, travel, food, water, home and child care among others regardless of the fact that most patients were poor and could hardly bear the costs, the patients expressed need for financial support to cater for these costs [7].

According to Aniebue [17], report on ethical, socioeconomic, and cultural considerations in gynecologic cancer care in South Africa, contrasting results were found. The report from South Africa revealed that cancer treatment was free for patients who earn less than an established minimum income. The same report also revealed that in India, surgery, radiotherapy, and chemotherapy treatments were partially or totally subsidized by government depending on a set minimum income level. Financial needs among cancer patients have also been reported by Sepulveda, Habiyambere [21] in their study conducted in a couple of African countries (Botswana, Ethiopia, Tanzania, Uganda and Zimbabwe) on quality of care at the end of life among cancer patients. Similarly, Murray, Grant [2] andMasika, Wettergren [7], also revealed need for financial support for care and treatment among cancer patients in Kenya and Tanzania respectively. Other international studies have also shown increasing need for financial assistance among cancer patients [22-24].

This finding shows that majority of the patients had a lot of pain, lacked energy and were forced to spend time in bed. More than half of the patients were bothered by constipation and nausea, this could be explained by the fact that most of the patients presented late with advanced disease with others suffering anaemia due to blood loss. Physical health issues among cancer patients have also been reported by other studies in Africa [2, 6, 7, 21, 25].

\section{Conclusion and Recommendations}

The present research illustrates unsatisfactory psychological, social, spiritual and physical well-being among cervical cancer patients. There is a need for the government to increase budgetary allocation for cancer management and explore modalities of subsidizing cervical cancer treatment to enable most patients to afford. The wider society also needs to be sensitized to offer support to members of the society suffering from life threatening diseases such as cervical cancer among them. Finally, more studies should be done focusing on wellbeing of patients suffering from other types of cancers.

\section{Competing Interests}

The author declares no competing interests.

\section{Funding}

This work was supported by kind contributions from family members.

\section{Acknowledgements}

We are indebted to cervical cancer patients in Western Kenya, the research assistants for data collection and JOOTRH staff for their assistance.

\section{References}

[1] NCCPP, Kenya National Cervical Cancer Prevention Program -Strategic Plan 2012-2015, M. o. P. H. a. S. a. M. o. M. services, Editor. 2012.

[2] Murray, S. A., et al., Dying from cancer in developed and developing countries: lessons from two qualitative interview studies of patients and their carers. BMJ, 2003. 326(7385): p. 368 .

[3] Ntizimira, C. R., et al., Palliative care in Africa: a global challenge. Ecancermedicalscience, 2014. 8: p. 493.

[4] Selman, E. L., Higginson, I. J., \& Harding, R., Quality of life among patients receiving palliative care in South Africa and Uganda: a multi-centred study. Health Qual Life Outcomes, 2011. 
[5] GOK, Cancer Prevention and Control Act 2012.

[6] Muiva, N. M., Assessment of the quality of life issues of women with gynaecological and breast cancers in Kenya., in Nursing Department. 2014, Nairobi University.

[7] Masika, G. M., et al., Health-related quality of life and needs of care and support of adult Tanzanians with cancer: a mixedmethods study. Health Qual Life Outcomes, 2012. 10: p. 133.

[8] Tadele, N., Evaluation of quality of life of adult cancer patients attending Tikur Anbessa specialized referral hospital, Addis Ababa Ethiopia. Ethiop J Health Sci, 2015. 25(1): p. 53-62.

[9] UNAIDS, United Nations Program on HIV/AIDS -UNAIDS report of the global AIDS epidemic 2013. 2013.

[10] KAIS, Kenya AIDS Indicator Survey 2014.

[11] Agius, P. A., et al., Human papillomavirus and cervical cancer: Gardasil vaccination status and knowledge amongst a nationally representative sample of Australian secondary school students. Vaccine, 2010. 28(27): p. 4416-22.

[12] Inzaule, S., et al., Incidence and predictors of first line antiretroviral regimen modification in western Kenya. PLoS One, 2014. 9(4): p. 93106.

[13] Kamau, R. K., A. O. Osoti, and E. M. Njuguna, Effect of diagnosis and treatment of inoperable cervical cancer on quality of life among women receiving radiotherapy at Kenyatta National Hospital. East Afr Med J, 2007. 84(1): p. 24-30.

[14] Ogoncho, I. M., Omuga, B. O., Wakasiaka, S., Muiva, M.,, Determinants of Quality of Life Among Gynaecological Cancer Patients on Follow Up at a Referral Hospital in Kenya. American Journal of Nursing Science, 2015. Vol. 4(No. 3, 2015): p. 127-130.

[15] Lamb, M. A., Psychosexual issues: the woman with gynecologic cancer. Semin Oncol Nurs, 1990. 6(3): p. 237-43.

[16] Ye, S., et al., A systematic review of quality of life and sexual function of patients with cervical cancer after treatment. Int J Gynecol Cancer, 2014. 24(7): p. 1146-57.

[17] Aniebue, U., U., \& Tonia, C. O., Ethical, Socioeconomic, and
Cultural Considerations in Gynecologic Cancer Care in Developing Countries International Journal of Palliative Care 2014.

[18] Rawlinson, F., et al., The current situation in education and training of health-care professionals across Africa to optimise the delivery of palliative care for cancer patients. Ecancermedicalscience, 2014. 8: p. 492.

[19] Betty, R., Ferrell, Karen, H. D., Quality of Life Among LongTerm Cancer Survivors. Oncology, 1997. 11 (4): p. 565-571

[20] Balboni, T. A., Paulk, M. E., Balboni, M. J., Phelps, A. C., Loggers, E. T., Wright, A. A., Block, S. D., Lewis, E. F., Peteet, J. R., and Prigerson, H. N., Provision of Spiritual Care to Patients With Advanced Cancer: Associations With Medical Care and Quality of Life Near Death. J Clin Oncol., 2010. 28( 3): p. 445-452.

[21] Sepulveda, C., et al., Quality care at the end of life in Africa. BMJ, 2003. 327(7408): p. 209-13.

[22] Arndt, V., et al., Quality of life in patients with colorectal cancer 1 year after diagnosis compared with the general population: a population-based study. J Clin Oncol, 2004. 22(23): p. 4829-36.

[23] Arndt, V., et al., Persistence of restrictions in quality of life from the first to the third year after diagnosis in women with breast cancer. J Clin Oncol, 2005. 23(22): p. 4945-53.

[24] Berkman, B. J. and S. E. Sampson, Psychosocial effects of cancer economics on patients and their families. Cancer, 1993. 72(9 Suppl): p. 2846-9.

[25] Harding, R. and I. J. Higginson, Palliative care in sub-Saharan Africa. Lancet, 2005. 365(9475): p. 1971-7.

[26] Sharma, K., et al., Pattern of palliative care, pain management and referral trends in patients receiving radiotherapy at a tertiary cancer center. Indian J Palliat Care, 2009. 15(2): p. 148-54.

[27] Abrahamson, K., Durham, M., Fox, R., Managing the unmet psychosocial and information needs of patients with cancer. Patient Intelligence 2010. 